\title{
Ineffectiveness for Infants of Immunization of Mothers with Pneumococcal Capsular Polysaccharide Vaccine During Pregnancy
}

\author{
Claudia R.C. Lopes ${ }^{1}$, Eitan N. Berezin ${ }^{2}$, Ting Hui Ching ${ }^{3}$, Jaildo de Souza Canuto ${ }^{4}$, Vanilda Oliveira da Costa ${ }^{4}$ and Érika \\ Monteiro Klering ${ }^{4}$ \\ ${ }^{1}$ Santa Casa de Misericórdia de São Paulo/HSLG; ${ }^{2}$ Pediatric Department, Santa Casa Medical School; ${ }^{3}$ Epidemiology Department, Santa Casa \\ Medical School; ${ }^{4}$ Clinical Laboratory, Santa Casa de Misericórdia de São Paulo/HSLG; São Paulo, SP, Brazil
}

\begin{abstract}
Pneumococcal (Pnc) carriage is associated with pneumococcal diseases. Breast feeding and maternal vaccination may be a useful approach to prevent pneumococcal infection in young infants. We examined the risk of Pnc carriage by infants at six months of age after pneumococcal polysaccharide vaccination of pregnant women. We selected 139 pregnant woman. The woman were randomly allocated to receive 23-valent polysaccharide vaccines during pregnancy (Group 1) after pregnancy (Group 2) or not receive any vaccine (Group 3). Nasopharyngeal swabs were collected from the infants at three and six months of age. The infants were evaluated monthly during the first six months. We included 47 mothers in Group 1, 45 mothers in Group 2 and 47 mothers in Group 3. Forty-seven percent of the babies were exclusively breast fed until six months, $26 \%$ received both breast feeding and artificial feeding and $13 \%$ received only artificial feeding. Among those patients, $26 \%$ were colonized by Pnc at six months (12 from Group 1, 13 from Group 2, and 12 from Group 3). There was no significant difference in colonization between the three groups. Thirty percent of the children were colonized by a non-susceptible strain. We concluded that young infants (three months old) are already susceptible to pneumococcal carriage. Vaccination during pregnancy with a polysaccharide vaccine did not decrease Pnc colonization.
\end{abstract}

Key-Words: Pneumococcal diseases, vaccine in pregnancy, colonization.

Pneumococcus (Pnc) infections are a leading cause of severe bacterial disease and are one of the most serious concerns for public health worldwide. It is estimated that Pnc causes more than one million deaths of children under five years old yearly, most of them by pneumonia [1-4]. Death rates can be estimated as two children per hour in Latin America [5]. Pneumococcal meningitis may reach 10\%-30\% mortality, with higher morbidity and mortality rates in children under six months of age [6,7].

Colonization of the upper respiratory tract is the first step towards infection. In Brazil, the nasopharyngeal colonization frequency is about $35 \%$ in children under two years old [811]. However, there are no studies of Pnc colonization rate in children younger that one year old. Some studies suggest that immune factors, namely anticapsular serum IgG can prevent colonization $[12,13]$.

The pneumococcal capsular polysaccharide capsule is an essential virulence determinant, and protection against invasive pneumococcal disease is correlated with specific serum antibody to this polysaccharide. A vaccine that contains the capsular polysaccharides of the 23 serotypes of $S$. pneumoniae has been licensed since 1983 for use in high-risk populations. However, this vaccine is poorly immunogenic in infants under two years of age. Pneumococcal glycoprotein conjugate vaccines are immunogenic in young infants, but the vaccine schedule initiates at two months and are unlikely to provide protection before a second dose at four to five

Received on 12 August 2008; revised 15 December 2008.

Address for correspondence: Dr. Claudia Lopes. E-mail: claudiarclopes@terra.com.br.

The Brazilian Journal of Infectious Diseases 2009;13(2):104-106. (C) 2009 by The Brazilian Journal of Infectious Diseases and Contexto Publishing. All rights reserved. months of age. Maternal immunization is a strategy with the potential to provide passive protection to infants during the vulnerable first few months of life, before active immunization of the infant is possible [14-18].

We evaluated the effect of maternal immunization against Pneumococcus using the 23-valent polysaccharide pneumococcal vaccine (PS-23), based on the frequency of Pnc nasopharyngeal colonization and on the rate of acute respiratory infections (ARI) related to S. pneumoniae.

\section{Material and Methods}

This study was performed at São Luis Gonzaga Hospital, managed by Santa Casa de Misericórdia de Sao Paulo. The Intern Review Board of Santa Casa de Sao Paulo Healthy approved the study. Pregnant women without significant obstetrical pathologies were included in this study from May 2005 to January 2006.

Afterproviding written informed consent, pregnant women were randomly assigned to one of three groups for the study. Group 1 receive no vaccine, Group 2 received PS-23 at 30 to 34 weeks of gestation and Group 3 received PS-23 after delivery.

The inclusion criteria were age 15-40 years, with an uncomplicated pregnancy and 30-34 gestation weeks; women should not have been at risk for complicated labor, and were without antecedents of a previous complicated pregnancy.

We interviewed every mother with emphasis to detect risk factors for pneumococcal disease, such as smoking at home, other children living in the house, and daycare frequency. During this visit, the mothers were questioned about adverse events of maternal immunization.

The infants were evaluated monthly during the first six months. During the visits the mothers were questioned about acute respiratory infections (ARI), such as acute obits media (AOM), pneumonia, fever, and other diseases potentially 
Table 1. Risk/protection factors for acute respiratory infection (ARI) among Group 1 (pregnant mother with not vaccinated), Group 2 (vaccine during pregnancy) and Group 3 (vaccine after delivery).

\begin{tabular}{lcccc}
\hline Risk or protection factors & Group 1 (47) & Group 2 (45) & Group 3 (47) & p \\
\hline Smoker living in household & $29.8 \%(14)$ & $48.9 \%(22)$ & $46.8 \%(22)$ & 0.12 \\
Siblings under five years old in household & $31.9 \%(15)$ & $33.3 \%(15)$ & $42.6 \%(20)$ & 0.50 \\
Exclusive breast milk feeding until six months & $47.8 \%(22)$ & $45.2 \%(19)$ & $53,3 \%(24)$ & 0.88 \\
More than five inhabitants at home & $27.7 \%(13)$ & $33.3 \%(15)$ & $27.7 \%(13)$ & 0.79 \\
Attending daycare & $12.8 \%(6)$ & $20 \%(9)$ & $17 \%(8)$ & 0.643 \\
\hline
\end{tabular}

Table 2 Nasopharyngeal pneumococcal colonization among Group 1 (no vaccine), Group 2 (vaccine during pregnancy) and Group 3 (vaccine after delivery).

\begin{tabular}{lccccc}
\hline & \multicolumn{1}{c}{ Total } & Group 1 & Group 2 & Group 3 & p \\
\hline Positive colonization at 3 months old & $17.3 \%(24 / 139)$ & $17 \%(8 / 47)$ & $22 \%(10 / 45)$ & $12.8 \%(6 / 47)$ & 0.486 \\
Positive colonization at 6 months old & $16.5 \%(22 / 133)$ & $15.2 \%(7 / 46)$ & $16.7 \%(7 / 42)$ & $17.8 \%(8 / 45)$ & 0.947 \\
Positive colonization at 3 or 6 months old & $26.6 \%(37 / 139)$ & $25.5 \%(12 / 47)$ & $28.9 \%(13 / 45)$ & $25.5 \%(12 / 47)$ & 0.916 \\
\hline
\end{tabular}

Table 3. Acute respiratory infection episodes and use of antibiotics until six months old, among Group 1 (no-vaccine), Group 2 (vaccine during pregnancy) and Group 3 (vaccine after delivery).

\begin{tabular}{lccccl}
\hline & Total & Group 1 & Group 2 & Group 3 & p \\
\hline ARI until 3 months old & $8.6 \%(12 / 139)$ & $8.5 \%(4 / 47)$ & $8.9 \%(4 / 45)$ & $8.5 \%(4 / 47)$ & 0.997 \\
ARI until 6 months old & $17.3 \%(23 / 133)$ & $17.4 \%(8 / 46)$ & $16.7 \%(7 / 42)$ & $17.8 \%(8 / 45)$ & 0.990 \\
Use of antibiotics until 6 months old $29.06 \%(40 / 139)$ & $25.5 \%(12 / 47)$ & $20 \%(14 / 45)$ & $29.8 \%(14 / 47)$ & 0.55 \\
\hline
\end{tabular}

caused by Pnc. The mother was also questioned about nutrition antecedents, breast feeding, and daycare center frequency.

Nasopharyngeal specimens were collected from the nose, using a calcium alginate swab and plated immediately after collection onto trypticose soy agar with $5 \%$ sheep blood and garamicin at $5 \mathrm{mcg} / \mathrm{mL}$ and transported to the microbiology laboratory within $4 \mathrm{~h}$. Specimens were inoculated in 5\% defibrinated sheep blood agar and incubated at $37^{\circ} \mathrm{C}$. Plates were read at 24 and 48 h. Streptococcus pneumoniae was identified by alpha-hemolytic activity, colony morphology and optochin disc susceptibility. Susceptibility of isolates to penicillin was determined by screening with a 1 mcg oxacillin disk [19].

The Chi-square test was used to compare the groups. A significant difference was considered when $\mathrm{p}<0.05$. EPI INFO software for Windows - version 13.0 was used to perform the analysis.

\section{Results}

One hundred and fifty women in the third trimester of pregnancy who met the inclusion criteria agreed to participate in the study. The mothers and infants in the two study groups were similar in both demographic and other characteristics (Table 1). There were 11 exclusions. Three patients were excluded due to gestation before one month after the vaccine (Group 2), one due to premature labor of 32 weeks in Group 1, and seven women left the study after signing the informed consent and being allocated (two in Group 1, two in Group 2, and three in Group 3). All remaining children were born after at least 37 weeks of pregnancy. The remaining 139 pregnant women were allocated as follows: 47 patients in Group 1 (34\%), 45 in Group 2 (32\%) and 47 in Group 3 (34\%).

There was no significant relationship between use of the vaccine and premature labor, which occurred in 2\% (1/50) of the women in Group 1, 6\% (3/50) in Group 2, and 0 in Group 3 $(p=0.184)$. No relevant adverse effects of the vaccine were detected in the 92 immunized patients in Groups 2 and 3. Only two pregnant women complained of local pain, and none developed fever.

Table 1 shows the distribution of patients according to risk or protection factors in each group, and compares groups. The mothers and infants in the three study groups were similar demographically and were considered homogeneous for the risk factors. The rate of pneumococcal nasopharyngeal colonization evaluated at the ages of three and six months old was similar in the three groups (Table 2). We isolated 37 pneumococcal strains. The rate of penicillin non-susceptible (PNS) Streptococci was 25\%; it was similar among the three groups. The rate of ARI was similar among the three groups (Table 3). None of the children developed invasive diseases during follow-up.

\section{Discussion}

We did not observe differences in ARI or Pnc colonization rate among the infants. Most studies of the use of pneumococcal vaccine in pregnant women evaluated antibodies and immunogenicity levels, without examining 
clinical data [20-23]. Studies by Carvalho [22] et al. and Shahid et al. [23] showed effective transplacental transfer of pneumococcal antibodies. Chaithongwongwatthana et al. [24] performed a meta-analysis of reports of pneumococcal maternal immunization; they did not find a decrease in the rate of ARI in infants of vaccinated mothers.

When we evaluated the colonization rate at three and six months, we found no other studies evaluating colonization rates in young infants. However, the colonization rate that we found was similar to previous findings, which revealed that colonization by pneumococcus can occur early in life, including colonization by PNS [10-12].

The three groups were homogeneous in terms of risk factors for pneumococcal disease, including breast-feeding, passive smoking, agglomeration, contact with other children, frequency in daycare centers and maternal education level. There were also no significant differences in colonization rate or ARI frequency in infants among the groups.

In conclusion, we found that Pneumococcus nasopharyngeal colonization rates did not change with immunization of pregnant or puerperal women with pneumococcal 23V-polysaccharide vaccine. The proposed intervention did not significantly alter the frequencies of diseases more commonly related to pneumococcal infection (ARI). We also demonstrated early S. pneumoniae colonization of infants.

\section{Acknowledgements}

The authors thank CNPq and the Fundo de Apoio a Pesquisa (FAP) da Faculdade de Ciencias Medicas da Santa Casa de São Paulo for support.

\section{References}

1. WHO. Etiology and clinical signs of serious infections in young infants in developing countries: a WHO collaborative study. Pediatr Infect Dis J 1999;18(suppl):561-9.

2. Klein J.O. The epidemiology of pneumococcal disease in infants and children. Rev Infect Dis 1981;3:246-53.

3. Black S.B, Shinefield H.R., Ling S., et al. Effectiveness of heptavalent pneumococcal conjugate vaccine in children younger than five years of age for prevention of pneumonia. Pediatr Infect Dis J 2002;21:810-5.

4. Gray R.M., Converse G.M. II, Dillon H.C. Jr. Epidemiologic studies of Streptococcus pneumoniae in infants: acquisition, carriage, and infection during the first 24 months of life. J Infect Dis 1980;142:923-33.

5. Jon Andrus. Second Regional Pneumococcal Symposium. December, 2006; PAHO (Pan American Health Organization).

6. Arditi M., Mason E.O. Jr., Bradley J.S., et al. Three-year multicenter surveillance of pneumococcal meningitis in children: clinical characteristics, and outcome related to penicillin susceptibility and dexamethasone use. Pediatrics 1998;102:1087-97.
7. Berezin E.N., Falleiros-Carvalho L.H., Lopes C.R., et al. [Pneumococcal meningitis in children: clinical findings, most frequent serotypes and outcome.]. J Pediatr (Rio J) 2002; 78:19-23.

8. Rey L.C., Wolf B., Moreira J.L., Verhoef J., Farhat C.K. [Nasopharyngeal isolates of S. pneumoniae from healthy carriers and children with pneumonia: colonization rates and antimicrobial susceptibility]. J Pediatr (Rio J) 2002;78:105-12.

9. Berezin E.N., Cardenuto M.D., Ferreira L.L., et al. Distribution of Streptococcus pneumoniae serotypes in nasopharyngeal carriage and in invasive pneumococcal disease in São Paulo, Brazil. Pediatr Infect Dis J 2007:26; 643-5.

10. Ferreira L.L., Carvalho E.S., Berezin E.N., Brandileone M.C. [Nasopharyngeal colonization and antimicrobial resistance of Streptococcus pneumoniae isolated from children with acute rhinopharyngitis]. J Pediatr (Rio J) 2001;77:227-34.

11. Bogaert D., Groot R., Hermans P.W. Streptococcus pneumoniae colonization: the key to pneumococcal disease. Lancet Infect Dis 2004;4:144-5.

12. Dagan R., Givon-Lavi N., Fraser D., et al. Serum serotype-specific pneumococcal anticapsular immunoglobulin g concentrations after immunization with a 9-valent conjugate pneumococcal vaccine correlate with nasopharyngeal acquisition of pneumococcus. J Infect Dis 2005;192:367-76.

13. Miller E.V., O’Brien K.L., Bronsdon M., et al. Anticapsular Serum antibody concentration and protection against pneumococcal colonization among children vaccinated with 7-valent pneumococcal conjugate vaccine Clin Infect Dis 2007;44:1173-9.

14. Greenwood B. Maternal immunization in developing countries. Vaccine 2003;21:3436-41.

15. Lehmann D., Pomat W.S., Combs B., et al. Maternal immunization with pneumococcal polysaccharide vaccine in the highlands of Papua New Guinea. Vaccine 2002;20:1837-45.

16. Mulholland K. Maternal immunizations for the prevention of bacterial infection in young infants. Vaccine 1998;16:1464-7.

17. Glezen W.P. Maternal vaccines. Prim Care 2001;28:791-803.

18. Halsey N.A, Klein D. Maternal immunization. Pediatr Infect Dis J 1990;9:574-81.

19. O’Brien K.L., Nohynek H. Report from a WHO Working Group: standard method for detecting upper respiratory carriage of Streptococcus pneumoniae. Pediatr Infect Dis J 2003;22:e1-11.

20. Englund J.A., Glezen W.P., Turner C., et al. Transplacental antibody transfer following maternal immunization with polysaccharide and onjugate Haemophilus influenza type b vaccines. J. Infect Dis 1995; $171: 99-105$.

21. Mulholland K., Suara R.O., Siber G.R., et al. Maternal immunization with Haemophilus influenza type b polysaccharide-tetanus protein conjugates vaccine in The Gambia. JAMA 1996;275:1182-8.

22. Carvalho. T., Caneiro-Sampaio M.M., Sole D., et al. Transplacental transmission of serotype-specific pneumococcal antibodies in a Brazilian population. Clin Diagn Lab Immunol 1999;6:50-4.

23. Shahid N.S., Steinhoff M.C., Hoque S.S., et al. Serum, breast milk, and infant antibody after maternal immunization with pneumococcal vaccine. Lancet 1995;346:1252-7.

24. Chaithongwongwatthana S., Yamasmit W., Limpongsanurak S., et al. Pneumococcal vaccination during pregnancy for preventing infant infection. Cochrane Database Syst Rev 2006;(1):CD004903. 